\title{
Success of a Tobacco Cessation Program for Parents at a Children's Hospital
}

\author{
Leighann Sweeney, Laura Taylor, Jubrae Peurifoy, Kelly Kauffman, and Natalie Napolitano
}

\begin{abstract}
BACKGROUND: Children are most affected by passive smoke and least able to avoid it. The Tobacco Dependence Program was developed to help reduce first-, second-, and thirdhand smoke exposure to our patients while they are in the hospital. METHODS: The Tobacco Dependence Program consists of a director, 3 coordinators, and 15 team members certified as tobacco treatment specialists. The program is offered to any admitted patient or his or her caregiver(s). We support participants whether their goal is to quit smoking or to abstain during the hospitalization. We provide weekly counseling and free over-the-counter nicotine replacement therapy for the entire duration of the hospitalization. The inclusion criterion for caregivers is a 7-d minimum stay. In-patients are automatically eligible. Before discharge, we provide a referral to an out-patient program. RESULTS: As of March 2019, we enrolled 138 participants, 5\% were in-patients. Fiftyfive percent had children who were critically ill and in the ICU: pediatric ICU/progressive care unit (PCU) 28\%, neonatal ICU 19\%, cardiac ICU (CICU) 9\%. The ethnicity of the participants was predominately white (56\%), followed by African American (33\%), Hispanic (12\%), and Asian $(<1 \%)$. Fifty-six percent of the participants were female. Fifty-nine percent of the participants began smoking between the ages of 12 and $18 \mathrm{y}, 21 \%$ began between the ages of 18 and $25 \mathrm{y}, 9 \%$ began before the age of $12 \mathrm{y}$, and $7 \%$ between the ages of 25 and $35 \mathrm{y}$. Fifty percent of the survey respondents stated that they were able to quit before discharge. CONCLUSIONS: The Tobacco Dependence Program offers support to families as they begin the cessation process and provide the tools to continue a smoke-free lifestyle after discharge. The program is feasible and welcomed to assist patients and families in this process. Key words: tobacco cessation; smoking cessation; pediatrics; counseling. [Respir Care 2020;65(4):407-412. (C) 2020 Daedalus Enterprises]
\end{abstract}

\section{Introduction}

Passive smoke exposure is known to cause impairment in the health of children, such as more frequent and severe asthma attacks, respiratory infections, ear infections, and sudden infant death syndrome in children. Hospital-

All authors are affiliated with Respiratory Therapy Department, Children's Hospital of Philadelphia, Philadelphia, Pennsylvania.

Financial Support: CVS Health.

Ms Napolitano discloses relationships with Aerogen, Draeger, Smiths Medical, Actuated Medical, and Vero Biotech. The remaining authors have disclosed no conflicts of interest.

Ms Taylor presented a version of this paper as an Editors' Choice abstract at the AARC Congress 2018, held December 4-7, 2018 in Las Vegas, Nevada. initiated tobacco cessation programs have been successful in adult hospitals but have yet to be initiated in pediatric institutions. Parents of children who are hospitalized are receptive to tobacco cessation support; however, inpatient pediatric clinicians rarely provide this option of parental support because it is not a reimbursable procedure. $^{1,2}$ The Respiratory Therapy Department at the Children's Hospital of Philadelphia recognized that there was a need for a tobacco cessation program to support

\footnotetext{
Supplementary material related to this paper is available at http://www. rcjournal.com

Correspondence: Laura Taylor MSc RRT RRT-NPS CTTS-M, Respiratory Care Department, 3401 Civic Center Blvd., Philadelphia, PA 19104.E-mail: taylorl6@email.chop.edu.
}

DOI: $10.4187 /$ respcare. 06810 


\section{Tobacco Cessation for Parents of Patients}

patients and their families. The program's objectives were to (1) help adolescent in-patients and caregivers abstain from smoking during a hospital admission, and (2) help adolescent in-patients and caregivers quit smoking. This is a report of the landscape of this new innovative program.

\section{See the Related Editorial on Page 575}

\section{Methods}

The Tobacco Dependence Program (TDP) was established to fill a recognized need for a tobacco cessation program to assist parents and patients to quit using tobacco or to abstain during admission. A 2-year grant was awarded from CVS Health to assist with startup costs of the program. Two patient populations that were the most affected by second- and thirdhand smoke exposure during admission were the asthma and neonatal populations. The most visible reaction was in other patients with asthma who were improving throughout their admission course and had visible setbacks when a parent or caregiver came in smelling like smoke.

When the TDP was initiated, it consisted of a director and a core team of 6 respiratory therapists. The program has now grown to 3 coordinators and 15 core team members who collectively screen, administer nicotine replacement therapies (NRT), and provide counseling to participants. Each program member has gone through training to become a certified tobacco treatment specialist. Children's Hospital of Philadelphia provides yearly training and education to TDP team members as well as the entire Respiratory Therapy Department to maintain competency and to provide the best experience for program participants. This provides them with the knowledge to assist with completing the initial screening process at the bedside. Analysis of the program from the Children's Hospital of Philadelphia Institutional Review Board was determined to be quality improvement and not human subjects research.

\section{Program Levels}

The TDP offers 2 levels of support. The initial objective is to help in-patients and caregivers abstain from using tobacco during their hospital course. The goal is that this will result in faster recovery times and shorter hospital stays. The second objective is to help in-patients and caregivers quit using tobacco entirely. The goal is that this will decrease readmission rates primarily for our population with asthma. The program is offered to any in-patient or any caregiver who lives in the patient's home and uses

\section{QUICK LOOK}

\section{Current knowledge}

Children are most affected by passive smoke and the least able to avoid it. The Tobacco Dependence Program (TDP) was developed to help reduce first-, second-, and thirdhand smoke exposure to our patients while they are admitted to the hospital. The TDP offers support to families as they begin the tobacco cessation process and provides the tools to continue a smoke-free lifestyle after discharge.

\section{What this paper contributes to our knowledge}

Lack of funding was one of the main obstacles during the creation and implementation of the TDP. However, the TDP had a large number of participants who drastically decreased their daily tobacco intake with support during the stressful time of having a child hospitalized. The success was achieved by offering free nicotine replacement therapies which removed the barrier of the financial cost of quitting and allowed the caregiver to focus on counseling.

tobacco. Weekly counseling, education, and free NRT are provided for the entire duration of the hospitalization. Although some program participants are adolescent patients and NRT is not FDA cleared for usage for individuals ages $<8 \mathrm{y}$, our physician group takes the stance of supporting cessation by using these medications. For parents and caregivers, a combination of over-the-counter NRT, including nicotine patches, nicotine gum, and nicotine lozenges in various dosages are available, and, for in-patients, the medical team orders the recommended NRT, and it is provided from the in-patient pharmacy. A strategic partnership with the PENN Harron Lung Center offers participants access to prescription NRT within close proximity to the hospital if needed. Weekly counseling focuses on the individual's stressors and triggers. Together, we come up with creative solutions for handling cravings and withdrawal symptoms.

The eligibility criterion for caregivers to participate in the TDP is a minimum hospitalization stay of $7 \mathrm{~d}$. The TDP defines a caregiver as any individual who will take part in the patient's care outside of the hospital. This could include parents, grandparents, siblings, or other family members or close friends. All in-patients, regardless of length of stay are eligible for the program. If a caregiver does not meet eligibility requirements, he or she is offered a referral to the quit line or a program in his or her residential state. In-patient asthma population has waived eligibility for parents and caregivers because the average stay for patients with asthma is $<7 \mathrm{~d}$ and is so sensitive to passive smoke exposure because it is often the cause of an 


\section{Tobacco Cessation for Parents of Patients}

exacerbation. The TDP also follows a unique population within our Special Delivery Unit of mothers carrying babies with known complex congenital conditions. These families are referred to us through their clinical team. At this time, we offer our services to not only the mothers but any individual who will be a caregiver in the child's life. The TDP follows these families through the entire pregnancy at their weekly out-patient visits and through delivery and the child's entire in-patient stay.

Before discharge the in-patients and caregivers are referred to out-patient and/or community programs through the American Lung Association Quit Line or the state or county department of health. A week after discharge, a member of the TDP calls the participant for a telephone follow-up counseling session. The final step in our follow-up procedure is an e-mail with a link that directs the participant to an anonymous questionnaire with regard to the services they received in our program. The information obtained from this questionnaire is critical to how we approach our participants and continue to improve our program.

\section{Program Flow Process}

During the admission process, bedside nurses obtain the patient's tobacco use or exposure history. If positive, the bedside nurse or medical team places a consult order to the TDP. A daily report is generated of in-patients with a current smoking status or exposure for screening in the event consult orders are not placed. A respiratory therapy staff member speaks with the tobacco users to determine interest in support for smoking cessation or abstinence and eligibility for the program, and makes notification to TDP or a referral to an out-patient program as appropriate according to the expected length of admission. If the patient or caregiver is interested in abstaining or quitting tobacco and meets the eligibility requirements, a TDP core team member will meet with him or her to complete the intake process. During the intake process, the TDP team member will ask for demographic information, medical history, current medication, and a history of tobacco use. The Fagerström Test for Nicotine Dependence (Fig. 1) is used to assess the participants' level of physical dependence to nicotine and to guide NRT recommendations. ${ }^{3}$ A sample of the program intake form used can be seen (in the supplementary materials at http://www.rcjournal.com).

Within the first few days of NRT usage, a counselor will check in with the participant to see if he or she has had any reactions or issues with the NRT. After a week of NRT use, the counselor will meet with the participant and discuss his or her progress and provide more NRT. During the counseling sessions, we work with the participant to creatively come up with different ways to abstain from using nicotine and discuss

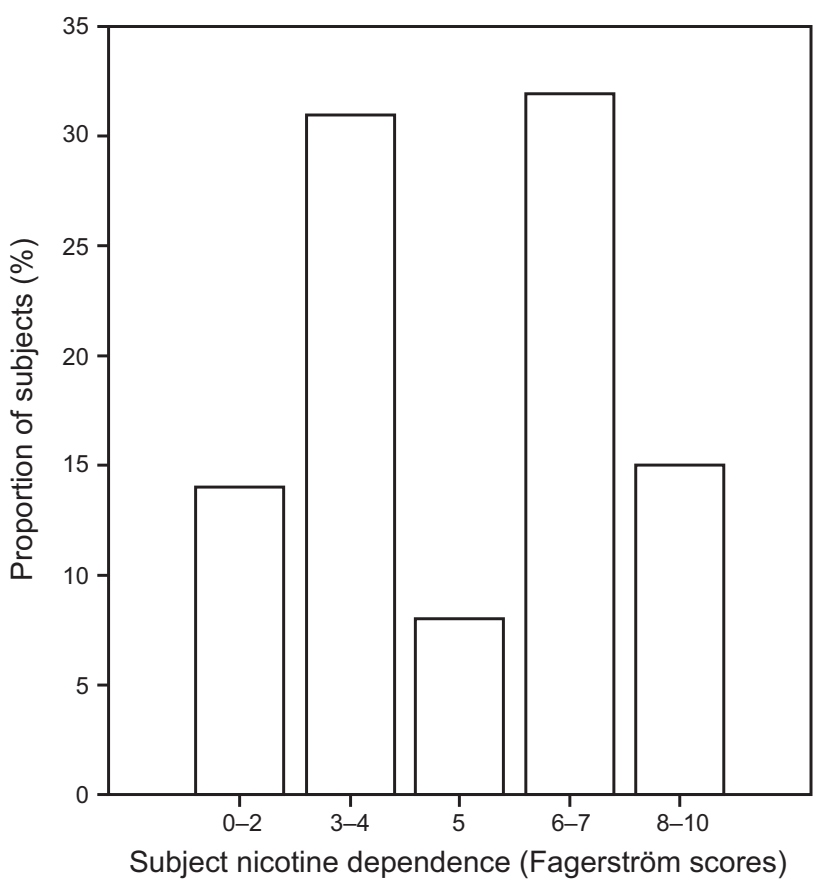

Fig. 1. Subject levels of nicotine dependence according to Fagerström scores.

stressors and personal challenges that the participant may face. We found that alternative methods can also help manage cravings. This could include anything from stress balls to exercise, meditation and yoga, journaling, or adult coloring. Additional ideas include changes to behavioral patterns. Descriptive summary statistics on program enrollment was tabulated with binomial distribution, and the chi-square test was used to analyze categorical data to determine any differences among groups of participants.

\section{Results}

As of March 2019, we enrolled 138 participants into the TDP. Demographics are shown in Table 1. Of these participants, 55\% (76) had children who were critically ill and in one of our ICUs. The pediatric ICU/PCU accounted for $28 \%$ (38) of the participants, followed by the neonatal ICU with $19 \%$ (26) and the CICU with $9 \%$ (12). The majority of TDP participants were caregivers of children in the ICU because these patients typically have a longer anticipated hospital length of stay and met the inclusion criteria due to illness. The participants were more commonly female $(56 \%)$ and were predominantly white $(56 \%)$, with their highest level of education being high school diploma/GED (46\%). On entry into the program, the median Fagerström score was 5 , and $93 \%$ of the participants stated they were ready to quit using tobacco; however, they were only somewhat confident they would be successful $(56 \%)$. Reasons 


\section{Tobacco Cessation for Parents of Patients}

Table 1. Demographics

\begin{tabular}{lc}
\hline \multicolumn{1}{c}{ Variable } & Result \\
\hline Female, $n(\%)$ & $77(56)$ \\
Age, median (range) y & $31(15-18)$ \\
Race/ethnicity, $n(\%)$ & \\
Caucasian & $77(56)$ \\
African American & $45(33)$ \\
Hispanic & $12(9)$ \\
Asian & $1(<1)$ \\
Other & $3(2)$ \\
Highest education level, $n(\%)$ & \\
High school/GED & $63(46)$ \\
Some high school & $21(15)$ \\
College degree & $17(12)$ \\
Some college & $33(24)$ \\
Graduate degree & $3(2)$ \\
Fagerström score, median (range) & $5(0-10)$ \\
ICU location, $n(\%)$ & $76(55)$ \\
\end{tabular}

Table 2. Reason for Quitting Tobacco Use

\begin{tabular}{lcc}
\hline \hline \multicolumn{1}{c}{ Reason } & $n(\%)$ & $P$ \\
\hline My health & $127(92)$ & $<.001^{*}$ \\
My family & $123(89)$ & $<.001^{*}$ \\
Stop smelling like smoke & $74(54)$ & .44 \\
Cost & $93(67)$ & $<.001^{*}$ \\
So my children won't smoke & $87(63)$ & $.003^{*}$ \\
Loved one is sick or died from smoking & $51(37)$ & .99 \\
& & \\
* Binomial distribution with no expected difference between the responses, and $P<.005$ was \\
considered significant.
\end{tabular}

for wanting to quit were collected on entry into the program, with the primary reason being for individual health and for their family (Table 2).

The response rate of the follow-up survey to date was $23 \%$ (32/138), which made it difficult to draw meaningful conclusions from the responses. The anonymous survey revealed that the participants were more likely to have a goal of reducing the amount of tobacco used or to quit smoking $(P=.005)$ on entry to the program and that, while in the program, they commonly changed to wanting to quit smoking $(P=.005)$. There was no difference in those reporting they wanted to quit smoking prior to hospital discharge $(P=.48)$. They predominantly rated their experience with the TDP as "excellent" or "very good" (84\%). The respondents of the follow-up survey often left positive comments about the program, such as "You guys have motivated and encouraged me to quit smoking," "I appreciate what the tobacco dependence program offers to parents while their child is sick, thank you so much!" and "The program has helped me a lot. I'm glad that this program is available to parents."

\section{Discussion}

We described the successful implementation of a tobacco cessation program in a children's hospital to support parents and caregivers as well as the adolescent patients. We showed that the majority of individuals in need at a children's hospital are the parents and caregivers and not the patient. This makes the funding of a program difficult because we are unable to obtain reimbursement for treatment of non-patients. The department and hospital leadership must recognize the importance of such a program and that the overall benefits will not be seen by the end of a hospital stay. The first 2 years of a startup are the most costly time of a program, and we were fortunate enough to obtain grant funding. With the outcomes and feedback from the first 2 years, the hospital has provided an increased budget within the Respiratory Therapy Department to continue this program long term, including the staff time, continuing education, and over-the-counter NRT. Although the postprogram survey return rate was low, the positive responses in the postprogram survey reinforced the need of this program.

Children who are exposed to environmental tobacco smoke are at increased risk for asthma, respiratory infections, and premature death. ${ }^{4-6}$ Parents who smoke put children at risk for adverse health outcomes and increase the chance that their children will become smokers. ${ }^{4-6}$ There have been limited studies that examined childhood hospitalization as a result of second-hand smoke exposure (SHS) smoke exposure. Thus, anything we, as their medical providers, can do to help reverse these trends should be done.

The American Academy of Pediatrics has a clinical policy against tobacco use and takes the stance that pediatricians should intervene with parents and caregivers who smoke to effect the outcomes of the child's health. ${ }^{7}$ The American Academy of Pediatrics recommends asking about tobacco use and smoke exposure during physician appointments. ${ }^{7}$ Physician recommendations toward treatment options should also be mentioned, and counseling should be provided to teens and young adults as prevention initiatives. The American Academy of Pediatrics also suggests that smoke exposure in utero can have long-lasting effects. ${ }^{7}$ The important stance the American Academy of Pediatrics takes is the foundation for the Children's Hospital of Philadelphia TDP program. ${ }^{7}$ Further focusing attention on the child's health versus the smoker's health has shown to be effective in helping parents quit smoking. ${ }^{2}$

Parents who smoke often see their child's physician more frequently than they see their own physician. It is vital to reach out to parents during these visits and express 


\section{Tobacco Cessation for Parents of Patients}

concern for environmental tobacco smoke exposure and what they can do to quit smoking. The Clinical Effort Against Secondhand Smoke Exposure program was created as a tool that primary care physicians can use to help parents quit smoking. ${ }^{8}$ Parents are screened, and those who smoke are given the Clinical Effort Against Secondhand Smoke Exposure action sheet that asks if he or she is interested in free cessation counseling or free NRT. Initial counseling begins with the child's physician and further counseling support is offered over the telephone. This would be helpful for our program because parents would already be screened and flagged as interested in further cessation support.

A comparison from the National Hospital Ambulatory Care Survey and the National Ambulatory Medical Care Survey from 1997-1999 and 2009-2011 was done to see progression in tobacco cessation counseling over the past decade. ${ }^{9}$ It was determined that there was a significant increase in counseling provided to pediatric patients age $<19$ y and parents by providers. The surveys examined various demographic information and tobacco use and/or exposure as well as if counseling was provided to the patient or parent. ${ }^{9}$ They showed an increase in tobacco counseling given to children ages $<19$ y from $1.5 \%$ in $1997-1999$ to $3.8 \%$ from $2009-2011 .{ }^{9}$ It was noted that counseling was more frequently provided during a well visit from the 2009-2011 time period than previously reported. The survey from 1997-1999 showed reporting of counseling at well visits to be $4.1 \%$ in contrast to the $11.1 \%$ reported in the 2009-2011 survey. ${ }^{9}$ This particular increase could be due to the Healthy People 2020 initiative. Healthy People 2020 is an initiative that aims to improve the health of all Americans. One of the Healthy People 2020 objectives is to reduce illness, disability, and death related to tobacco use and secondhand smoke exposure. The reduction of tobacco advertising to children and their access to cessation is highlighted with the Healthy People 2020 objective. ${ }^{10}$ These should not only be out-patient goals, when the child is admitted to the hospital is an ideal time for a behavior change touchpoint and spend intensive time with tobacco counseling for success. An ideal program spans the continuum of care.

The Stop Tobacco Outreach Program implemented a smoking cessation intervention for parents who had a child hospitalized for a respiratory illness that included an initial motivational interview, written education materials, NRT, telephone counseling, and a fax referral to the parents' primary clinician. ${ }^{11}$ This study also engaged parents while their child was hospitalized and had also shown parents were receptive and wanted support. ${ }^{11}$ The main difference with our program was that the participant's referrals are to a local cessation program instead of their primary care physician. There is no guarantee that their primary care physician is knowledgeable on tobacco cessation treatment and is able to appropriately continue to support this.

\section{Limitations}

Several obstacles were recognized over the course of creating and implementing the TDP. The first obstacle encountered was a lack of funding. Funding of a program of this kind is complicated because reimbursement could not be received as part of the hospital stay because we were predominantly treating the parents and caregivers and not the patient. The need for education and cessation programs in pediatric populations continues to grow; however, at this time, funding is limited. Grant funding is generally limited to program startup and is limited for continued management of a program. Accessibility of parents and caregivers is challenging. During hospital admissions, parents and caregivers are not always at the bedside due to the needs of other siblings or family members and work requirements. In many instances, intakes had to be completed over the telephone, which can make it difficult to form a connection and trust with the parent or caregiver.

Enrollment of adolescent in-patients was difficult due to a fear of reprimand from parents. It was of the upmost importance that confidentiality was maintained from the initial screening to discharge. Allowing these adolescents to speak freely without worry of penalty allowed us to work very closely in following up NRT adverse effects and helping develop creative strategies for dealing with peer pressure and withdrawal symptoms. These are topics that are often overlooked because the primary education toward adolescents at this time is to never begin smoking, not what to do when you have already begun. Small numbers within the program and the low response rate of the postdischarge survey make statistical analysis of the effectiveness of the program difficult.

\section{Future Goals}

Our future goals include increasing in-patient recruitment and response rate of the post discharge survey. We would also like to continue to broaden the resources available for adolescents to be successful in their tobacco cessation journey, including the development of out-patient support. There is also a need for long-term population health research to document the improvements in health outcomes of children when their passive smoke exposure decreases or is eliminated.

\section{Conclusions}

The development of a tobacco cessation program for patients and caregivers at a children's hospital is feasible welcomed and has the ability to help reduce tobacco intake during the stressful time of having a child hospitalized. 


\section{Tobacco Cessation for Parents of Patients}

\section{REFERENCES}

1. Winickoff JP, Hibberd PL, Case B, Sinha P, Rigotti NA. Child hospitalization: an opportunity for parental smoking intervention. Am J Prev Med 2001;21(3):218-220.

2. Rosen LJ, Noach MB, Winickoff JP, Hovell MF. Parental smoking cessation to protect young children: a systematic review and metaanalysis. Pediatrics 2012;129(1):141-152.

3. Heatherton TF, Kozlowski LT, Frecker RC, Fagerström KO. The Fagerström Test for nicotine dependence: a revision of the Fagerström Tolerance Questionnaire. Br J Addict 1991;86(9):119127.

4. USDHHS: Health consequences of involuntary exposure to tobacco smoke: a report of the Surgeon General. Washington, DC: US Government Printing Office; 2006. Available at: http://www. gaspforair.org/gasp/gedc/pdf/SurgeonGeneralSummary06.pdf. Accessed May 6, 2019.

5. Millett C, Lee JT, Laverty AA, Glantz SA, Majeed A. Hospital admissions for childhood asthma after smoke-free legislation in England. Pediatrics 2013;131(2):e495-e501.
6. Howrylak JA, Spanier AJ, Huang B, Peake RW, Kellogg MD, Sauers $\mathrm{H}$, Kahn RS. Cotinine in children admitted for asthma and readmission. Pediatrics 2014;133(2):e355-e362.

7. Farber HJ, Walley SC, Groner JA, Nelson KE; Section on Tobacco Control. Clinical practice policy to protect children from tobacco, nicotine, and tobacco smoke. Pediatrics 2015;136(5):1008-1017.

8. Winickoff JP, Park ER, Hipple BJ, Berkowitz A, Vieira C, Friebely $\mathrm{J}$, et al. Clinical effort against secondhand smoke exposure: development of framework and intervention. Pediatrics 2008;122(2): e363-e375.

9. Cawkwell PB, Lee L, Shearston J, Sherman SE, Weitzman M. The difference a decade makes: smoking cessation counseling and screening at pediatric visits. Nov 18, 2016. Available at: https://www.ncbi.nlm. nih.gov/pmc/articles/PMC5055743/. Accessed October 24, 2018.

10. Tobacco use. (n.d.). Available at: https://www.healthypeople.gov/ 2020/topics-objectives/topic/tobacco-use. Accessed October 25, 2018.

11. Winickoff JP, Hillis VJ, Palfrey JS, Perrin JM, Rigotti NA. A smoking cessation intervention for parents of children who are hospitalized for respiratory illness: the stop tobacco outreach program. Pediatrics 2003;111(1):140-145.

This article is approved for Continuing Respiratory Care Education credit. For information and to obtain your CRCE

(free to AARC members) visit

www.rcjournal.com 